\title{
C1r/C1s Deficiency
}

National Cancer Institute

\section{Source}

National Cancer Institute. C1r/C1s Deficiency. NCI Thesaurus. Code C119991.

Lack of production of either functional C1r or C1s protein, due to a genetic defect.

Approximately $60 \%$ of patients with a C1r/C1s deficiency will develop a severe systemic lupus erythematosus at an early age. Patients also present with frequent sinopulmonary infections often with Streptococcus pneumoniae. 